\title{
CONVERGENCE AND STABILITY FOR A HYPERBOLIC DIFFERENCE EQUATION
} WITH ANALYTIC INITIAL-VALUES

\author{
GERMUND DAHLQUIST
}

1. One of the most powerful methods for the numerical solution of partial differential equations is the method of finite differences. It is, however, well known that this method has to be used with some care. The problems occurring in actual practice are often very complicated, and a rigorous discussion of the relation of the solution of the given differential equation to the solution of the corresponding difference equation is in general very difficult. However, the investigation of simple cases, where both the differential equation and the difference equation can be solved explicitly, frequently shows some interesting features.

In this paper we shall consider the following classical problem: Given the functions $f(x)$ and $g(x)$ for $a \leqq x \leqq b$, find $F(x, t)$, satisfying the equations

$$
\begin{gathered}
\frac{\partial^{2} F}{\partial t^{2}}=\frac{\partial^{2} F}{\partial x^{2}} \\
F(x, 0)=f(x), \quad\left(\frac{\partial F}{\partial t}\right)_{t=0}=g(x) .
\end{gathered}
$$

This problem will be called "Problem $D$ " in contrast to the following similar problem for a difference equation, which will be called "Problem $\triangle$ ": Given the functions $f(x)$ and $g(x)$ for $a \leqq x \leqq b$. Find $\Phi(x, t)$, for $t=0, \pm \Delta t, \pm 2 \Delta t, \ldots$ satisfying the equations

$$
\begin{gathered}
\frac{\Phi(x, t+\Delta t)-2 \Phi(x, t)+\Phi(x, t-\Delta t)}{(\Delta t)^{2}}=\frac{\Phi(x+\Delta x, t)-2 \Phi(x, t)+\Phi(x-\Delta x, t)}{(\Delta x)^{2}}, \\
\Phi(x, 0)=f(x), \quad \Phi(x, \Delta t)=f(x)+\Delta t g(x),
\end{gathered}
$$

where $\Delta x$ and $\Delta t$ are constant, positive, finite increments. Note that $x$ is a continuous variable, while $t$ is a discrete variable.

It is well known that the solution of Problem $D$ is uniquely determined in the square

Received January 15, 1954. 


$$
a+|t| \leqq x \leqq b-|t|,
$$

that is called the region of determination of Problem $D$. The explicit solution is

$$
F(x, t)=\frac{1}{2}(f(x+t)+f(x-t))+\frac{1}{2} \int_{x-t}^{x+t} g(u) d u .
$$

In Problem $\Delta$ we get a recursive determination of $\Phi(x, t)$ in the rhombus determined by the inequalities

in which $u=\Delta x / \Delta t$.

$$
a+u|t| \leqq x \leqq b-u|t|,
$$

For convenience, we introduce certain linear operators acting on functions of $x$. Let $A_{t}[f(x)]$ be the solution of Problem $D$, when $g(x) \equiv 0$, and let $\alpha_{t}[f(x)]$ be the corresponding solution of Problem $\Delta$. From (1.3) it follows that

$$
A_{t}[f]=\frac{1}{2}(f(x+t)+f(x-t)) .
$$

The explicit expression for $\alpha_{t}[f]$ will be given in Section 6 . It is more complicated and is not convenient for our purpose.

Let $B_{t}[g(x)]$ be the solution of Problem $D$, when $f(x) \equiv 0$, and let $\beta_{t}[g(x)]$ be the corresponding solution of Problem $\Delta$. We have

$$
\frac{\partial B_{t}[g]}{\partial t}=A_{t}[g], \quad \frac{\beta_{t}[g]-\beta_{t-\Delta t}[g]}{\Delta t}=\alpha_{t}[g],
$$

since both sides of each equation are solutions of Problem $D$ and $\Delta$, respectively, satisfying the same initial conditions. For the difference equation this follows by means of the relation $\beta_{\Delta t}[g]+\beta_{-\Delta t}[g]=0$.

We shall study the following convergence problem. Let $\Delta x$ and $\Delta t$ tend to zero in such a way that $u=\Delta x / \Delta t$ remains constant. Is it true that $\Phi(x, t)$ tends to $F(x, t)$ uniformly in a certain region of the $x t$-plane? Courant et al. [2, p. $61 \mathrm{ff}$.] proved in 1928 that $\Phi(x, t) \rightarrow F(x, t)$ in the region of determination, if $u \geqq 1$, provided that $f^{\prime \prime \prime}(x)$ and $g^{\prime \prime}(x)$ are continuous for $a \leqq x \leqq b$. They pointed out, however, that if $u<1, \Phi$ does not tend to $F$ in general, in spite of the fact that $F(x, t)$ satisfies Problem $\Delta$ with an error that is $O\left((\Delta t)^{2}\right)$. The reason for this is that $\Phi\left(x^{\prime}, t^{\prime}\right)$ depends on the initial data of the segment $x^{\prime}-u t^{\prime} \leqq x \leqq x^{\prime}+u t^{\prime}$ only, whereas $F\left(x^{\prime}, t^{\prime}\right)$ depends on the initial values in the larger segment $x^{\prime}-t^{\prime} \leqq x \leqq x^{\prime}+t^{\prime}$. Thus, if we disturb $f(x)$ in the interval $x^{\prime}+u t^{\prime}<x \leqq x^{\prime}+t^{\prime}$ without disturbing it for $x \leqq x^{\prime}+u t^{\prime}$, then $F\left(x^{\prime}, t^{\prime}\right)$ will be changed, whereas $\Phi\left(x^{\prime}, t^{\prime}\right)$ will not be changed, however small $\Delta t$ has been chosen. This argument shows that we cannot have convergence in general. How- 
ever, it does not tell whether there is convergence or not for a given pair of functions $f(x), g(x)$. If $f(x)$ is analytic in the interval $a \leqq x \leqq b$, the basis of the argument does not hold, since one cannot change $f(x)$ for $x>x^{\prime}+u t^{\prime}$ without disturbing it for $x \leqq x^{\prime}+u t^{\prime}$.

The purpose of this paper is to study the convergence problem for analytic initial-values, when $u=\Delta x / \Delta t$ is constant, $u<1$. The main result is the following

TheOREm 1. Suppose that $f(x)$ and $g(x)$ can be extended to functions $f(z)$ and $g(z)$ of $z=x+i y$, which are holomorphic within and on the rectangle

$$
a \leqq x \leqq b, \quad|y| \leqq(b-a) / 3 .
$$

Let $\Delta t \rightarrow 0$ and $\Delta x \rightarrow 0$, in such a manner that $u=\Delta x / \Delta t$ remains constant . Then, even if $0<u<1$, the solution of Problem $\Delta$ converges to the solution of Problem $D$ uniformly in the square

$$
a+|t| \leqq x \leqq b-|t|
$$

in the real $x$-plane. If $u>5 / 9$, then the coefficient $1 / 3$ may be replaced by the smaller value $\frac{1}{2} u \operatorname{arcosh} u^{-1}$.

Since

$$
F(x, t)=A_{t}[f]+B_{t}[g], \quad \Phi(x, t)=\alpha_{t}[f]+\beta_{t}[g],
$$

we conclude by using (1.5) that it is sufficient to prove that $\alpha_{t}[f]$ tends to $A_{t}[f]$ uniformly. The proof of Theorem 1 will be completed in Section 5 where also, in Theorem 2, a generalization will be given. Section 4 contains an example in which a non-real singularity causes divergence. In Section 6 the relation of the convergence problem to the problem of numerical stability will be discussed briefly.

2. In Sections 2 and 3 we assume for simplicity that $t>0$. By symmetry the results can be extended to negative values of $t$.

Let us first seek solutions to (1.1) of the form

$$
\Phi(x, t)=e^{i(\lambda x+\gamma t)},
$$

$\lambda$ and $\gamma$ being complex numbers. We easily find the necessary and sufficient condition

$$
\frac{e^{i \gamma \Delta t}-2+e^{-i \gamma \Delta t}}{(\Delta t)^{2}}=\frac{e^{i \lambda \Delta x}-2+e^{-i \lambda \Delta x}}{(\Delta x)^{2}},
$$

or

$$
\sin \frac{1}{2} \gamma \Delta t= \pm u^{-1} \sin \frac{1}{2} \lambda \Delta x,
$$


which gives the relation between the speed and the frequency of "waves" in Problem 4 . (For Problem $D$, the speed is obviously independent of the frequency.) $\gamma$ is not determined uniquely by (2.1). However, if the complex $\frac{1}{2} \lambda \Delta x$-plane is cut along the real axis from $\arcsin u$ to $+\infty$ and from $-\arcsin u$ to $-\infty$, a one-valued branch is determined by the condition that $\gamma \Delta t \sim \lambda \Delta x / u$ when $\lambda \Delta x \rightarrow 0$. On the real axis, $\gamma$ shall be determined by a passage to the limit from the upper half-plane if $\operatorname{Re} \lambda>0$, and from the lower half-plane if $\operatorname{Re} \lambda<0$.

It is now easily verified that if $\gamma$ is determined by $\lambda$ according to (2.1) as just described, then

$$
\alpha_{t}\left[e^{i \lambda x}\right]=e^{i \lambda x} \cos \left(\gamma\left(t-\frac{1}{2} \Delta t\right)\right) / \cos \frac{1}{2} \gamma \Delta t .
$$

The corresponding relation in Problem $D$ is

$$
A_{t}\left[e^{i \lambda x}\right]=e^{i \lambda x} \cos \lambda t .
$$

Let $\Delta t \rightarrow 0$, while $x, t$ and $\lambda$ are kept fixed; observe that $u$ is constant during all limit processes. We infer then from the last three equations that $\gamma \rightarrow \lambda$ and that $\alpha_{t}\left[e^{i \lambda x}\right] \rightarrow A_{t}\left[e^{i \lambda x}\right]$. The convergence is moreover uniform in any closed, bounded region in $(\lambda, x, t)$-space. From these results we infer that $\alpha_{t}[f] \rightarrow A_{t}[f]$ for integral functions $f$ of exponential type, since we can use representations like

$$
f(x)=\int_{C} e^{i \lambda x} h(\lambda) d \lambda, \quad \alpha_{t}[f]=\int_{C} \alpha_{t}\left[e^{i \lambda x}\right] h(\lambda) d \lambda,
$$

where $C$ is a closed contour in the $\lambda$-plane and $h(\lambda)$ is continuous on $C$. (Cf., for example, Titchmarsh [5, Theorem 33].)

On page 92 an argument of Courant et al. was quoted from which we may infer that this result cannot be extended to the general class of functions that can be represented by Fourier integrals, for example, from $-\infty$ to $+\infty$ along the real axis. The explanation is, roughly speaking, that we have then to consider such $\lambda$ for which $\lambda \Delta x$ is considerable, even if $\Delta x$ is small. From (2.1) we see that if $\lambda$ is real and if $\left|\sin \frac{1}{2} \lambda \Delta x\right|>u$, then $\frac{1}{2} \gamma \Delta t=\frac{1}{2} i \gamma^{\prime \prime} \Delta t+\frac{1}{2} \pi+n \pi$, where $\gamma^{\prime \prime}$ is real and $n$ is an integer, and $\left|\frac{1}{2} \gamma^{\prime \prime} \Delta t\right|$ varies from 0 to $\operatorname{arcosh} u^{-1}$, which is real since $0<u<1$. Hence, in this case

$$
\alpha_{t}\left[e^{i \lambda x}\right]=-(-1)^{t / \Delta t} e^{i \lambda x} \sinh \left(\frac{1}{2} \gamma^{\prime \prime} \Delta t(2 t / \Delta t-1)\right) / \sinh \frac{1}{2} \gamma^{\prime \prime} \Delta t
$$

This diverges very rapidly, when $\Delta t$ approaches 0 , while $x, t$ and $\frac{1}{2} \lambda \Delta x$ are kept constant. This circumstance may spoil the convergence of $\alpha_{t}[f]$ to $A_{t}[f]$, unless $h(\lambda)$ behaves in a suitable manner for large $|\lambda|$. In 
Section 3, we shall see that the Fourier integral method may be applied with success to the case $f(x)=(x-\xi)^{-1}$. In Section 5, we shall extend the results to general analytic functions, by means of Cauchy's theorem.

We shall need a few inequalitites. Put

$$
\lambda=\lambda^{\prime}+i \lambda^{\prime \prime}, \quad \gamma=\gamma^{\prime}+i \gamma^{\prime \prime} .
$$

If $|p| \leqq M,|q| \leqq M$ and if $n$ is an integer, we have

$$
\left|\frac{p^{2 n+1}+q^{2 n+1}}{p+q}\right|=\left|p^{2 n}-q p^{2 n-1} \pm \ldots+q^{2 n}\right| \leqq(2 n+1) M^{2 n} .
$$

Put $t=(n+1) \Delta t, p=e^{\frac{1}{2} i \gamma \Delta t}, q=e^{-\frac{1}{2} i \gamma \Delta t}$. Then, by (2.2),

$$
\left|\alpha_{t}\left[e^{i \lambda x}\right]\right|=\left|\frac{p^{2 n+1}+q^{2 n+1}}{p+q}\right| e^{-\lambda^{\prime \prime} x} \leqq(2 t / \Delta t) e^{\left|\gamma^{\prime \prime}\right|(t-\Delta t)-\lambda^{\prime \prime} x} .
$$

From (2.3) we immediately obtain

$$
\left|A_{t}\left[e^{i \lambda x}\right]\right| \leqq e^{\left|\lambda^{\prime \prime}\right| t-\lambda^{\prime \prime} x} .
$$

When $|\lambda \Delta t|<1$, we may obtain the very crude inequality,

$$
|\gamma \Delta t-\lambda \Delta t|<|\lambda \Delta t|^{3},
$$

by an elementary application of Taylor expansions to (2.1) using dominant geometric series. Let us introduce $\eta=(\Delta t)^{\frac{1}{2}}$. If $\eta<1$ and if $|\lambda|<\eta^{-1}$, then

$$
|\lambda \Delta t|<\eta, \quad|\gamma \Delta t|<2 \eta, \quad|\gamma-\lambda|<\eta .
$$

Assume that $\lambda$ is real and that (2.7) holds. Then we conclude from (2.1) that $\gamma$ is real, and we get

$$
\begin{aligned}
\left|A_{t}\left[e^{i \lambda x}\right]-\alpha_{t}\left[e^{i \lambda x}\right]\right| & =\left|\cos \lambda t-\cos \gamma t-\operatorname{tg}\left(\frac{1}{2} \gamma \Delta t\right) \sin \gamma t\right| \\
& <|\gamma-\lambda| t+2\left|\frac{1}{2} \gamma \Delta t\right|<(t+2) \eta .
\end{aligned}
$$

Next, assume that $\lambda$ is real, but that (2.7) need not be satisfied. Put $u^{-1}\left|\sin \frac{1}{2} \lambda \Delta x\right|=\tau$. Then, by $(2.1)$

$$
\left|\frac{1}{2} \gamma^{\prime \prime} \Delta t\right|=|\operatorname{Im}(\arcsin \tau)|=\left\{\begin{array}{lll}
0 & \text { if } & \tau<1, \\
\operatorname{arcosh} \tau, & \text { if } & \tau>1,
\end{array}\right.
$$

whence we obtain

$$
\left|\gamma^{\prime \prime}\right| \leqq(2 / \Delta t) \max |\operatorname{arcosh} \tau|=(2 / \Delta t) \operatorname{arcosh} u^{-1} .
$$

We also need another estimate for $\gamma^{\prime \prime}$. From the definition of $u$ and $\tau$ follows $\left|\frac{1}{2} \lambda \Delta t\right|=\left|u^{-1} \frac{1}{2} \lambda \Delta x\right|>\tau$, and thus according to $(2.10)$

$$
\left|\gamma^{\prime \prime}\right| \lambda \mid \leqq \tau^{-1} \operatorname{arcosh} \tau \quad \text { for } \quad 1 \leqq \tau \leqq u^{-1} .
$$


The function $\tau^{-1} \operatorname{arcosh} \tau$ is increasing, until it reaches its maximum, somewhat less than $2 / 3$, when $\tau$ is a little larger than $9 / 5$. Thus we obtain

$$
\max \left|\gamma^{\prime \prime}\right| \lambda \mid=K \leqq\left\{\begin{array}{l}
2 / 3, \\
u \operatorname{arcosh} u^{-1} .
\end{array}\right.
$$

Finally, assume that $\lambda$ is imaginary. Put $\lambda=i \lambda^{\prime \prime}$. Then

$$
\sinh \frac{1}{2} \gamma^{\prime \prime} \Delta t=u^{-1} \sinh \frac{1}{2} \lambda^{\prime \prime} u \Delta t,
$$

whence $\left|\gamma^{\prime \prime}\right|<\left|\lambda^{\prime \prime}\right|$, because $u<1$. Then we can derive from (2.2)

$$
\left|\alpha_{t}\left[e^{-\lambda^{\prime \prime} x}\right]\right| \leqq e^{\left|\gamma^{\prime \prime}\right| t-\lambda^{\prime \prime} x} \leqq e^{\left|\lambda^{\prime \prime}\right| t-\lambda^{\prime \prime} x} .
$$

3. We shall consider the case $f(x)=(x-\xi)^{-1}$, where $\xi=\xi^{\prime}+i \xi^{\prime \prime}$ is a complex constant. To begin with, we assume that $\xi^{\prime \prime}<0$. Then we have

and

$$
(x-\xi)^{-1}=-i \int_{0}^{\infty} e^{-i \lambda \xi} e^{i \lambda x} d \lambda
$$

$$
\begin{aligned}
& A_{t}\left[(x-\xi)^{-1}\right]=-i \int_{0}^{\infty} e^{-i \lambda \xi} A_{t}\left[e^{i \lambda x}\right] d \lambda, \\
& \alpha_{t}\left[(x-\xi)^{-1}\right]=-i \int_{0}^{\infty} e^{-i \lambda \xi} \alpha_{t}\left[e^{i \lambda x}\right] d \lambda,
\end{aligned}
$$

since, by (2.6) and (2.5), we are allowed to perform operations under the integral signs in order to see that the equations and initial conditions for $A_{t}\left[(x-\xi)^{-1}\right]$, and $\alpha_{t}\left[(x-\xi)^{-1}\right]$ are satisfied. Hence

$$
\begin{aligned}
& \quad\left|A_{t}\left[(x-\xi)^{-1}\right]-\alpha_{t}\left[(x-\xi)^{-1}\right]\right| \\
& \leqq \int_{0}^{L} e^{-\lambda\left|\xi^{\prime \prime}\right|}\left|A_{t}\left[e^{i \lambda x}\right]-\alpha_{t}\left[e^{i \lambda x}\right]\right| d \lambda+\int_{L}^{\infty} e^{-\lambda\left|\xi^{\prime \prime}\right|}\left|A_{t}\left[e^{i \lambda x}\right]\right| d \lambda+\int_{L}^{\infty} e^{-\lambda\left|\xi^{\prime \prime}\right|}\left|\alpha_{t}\left[e^{i \lambda x}\right]\right| d \lambda \\
& \text { Put } \Delta t=\eta^{2} \text { and } L=\eta^{-1} \text {. Then, by (2.8), we get, }
\end{aligned}
$$

$$
I_{1}<\left|\xi^{\prime \prime}\right|^{-1}(t+2) \eta
$$

and by (2.6), (2.5) and (2.10), remembering that $\lambda^{\prime \prime}=0$ and $\xi^{\prime \prime}<0$,

$$
I_{2}<\left|\xi^{\prime \prime}\right|^{-1} e^{-\left|\xi^{\prime \prime}\right| / \eta}, \quad I_{3}<2 t \eta^{-2}\left|\xi^{\prime \prime}+K t\right|^{-1} e^{\left(\xi^{\prime \prime}+K t\right) / \eta} .
$$

These inequalities show that for any given positive values of $\varepsilon, \delta$ and $T$, a value of $\Delta t$ (or $\eta$ ) can be found, such that 


$$
\left|A_{t}\left[(x-\xi)^{-1}\right]-\alpha_{t}\left[(x-\xi)^{-1}\right]\right|<\varepsilon
$$

for all $x, t, \xi$ satisfying the relations

$$
0 \leqq t \leqq T, \quad \xi^{\prime \prime} \leqq-K t-\delta .
$$

The case $\xi^{\prime \prime}>0$ may be reduced to the case already considered by taking complex conjugates. Hence the same results hold more generally for

$$
0 \leqq t \leqq T, \quad\left|\xi^{\prime \prime}\right| \geqq K t+\delta .
$$

We shall obtain a different sufficient condition by the use of the following integral, analogous to (3.2)

$$
\alpha_{t}\left[(x-\xi)^{-1}\right]=\int_{0}^{\infty} e^{\lambda^{\prime \prime} \xi} \alpha_{t}\left[e^{-\lambda^{\prime \prime} x}\right] d \lambda^{\prime \prime},
$$

derived from the relation

$$
(x-\xi)^{-1}=\int_{0}^{\infty} e^{-\lambda^{\prime \prime}(x-\xi)} d \lambda^{\prime \prime} .
$$

From (2.12) it follows that $\gamma^{\prime \prime} \sim u \lambda^{\prime \prime}$, when $\lambda^{\prime \prime} \rightarrow \infty$. Hence (3.4) holds for $0 \leqq t<\left(x-\xi^{\prime}\right) / u$, whereas the corresponding formula for $A_{t}$ only holds for $0 \leqq t<x-\xi^{\prime}$. Assume that

$$
0 \leqq t<x-\xi^{\prime}-\delta, \quad|x| \leqq C, \quad|\xi| \leqq C .
$$

We have

$$
\begin{gathered}
\left|A_{t}\left[(x-\xi)^{-1}\right]-\alpha_{t}\left[(x-\xi)^{-1}\right]\right| \leqq \\
\int_{0}^{R} e^{\lambda^{\prime \prime} \xi^{\prime}}\left|A_{t}\left[e^{-\lambda^{\prime \prime} x}\right]-\alpha_{t}\left[e^{-\lambda^{\prime \prime} x}\right]\right| d \lambda^{\prime \prime}+\int_{R}^{\infty} e^{\lambda^{\prime \prime} \xi^{\prime}}\left|A_{t}\left[e^{-\lambda^{\prime \prime} x}\right]\right| d \lambda^{\prime \prime}+\int_{R}^{\infty} e^{\lambda^{\prime \prime} \xi^{\prime}}\left|\alpha_{t}\left[e^{-\lambda^{\prime \prime} x}\right]\right| d \lambda^{\prime \prime} \\
=I_{1}+I_{2}+I_{3} .
\end{gathered}
$$

Now, given any $\varepsilon>0$, we can choose a value of $R$, depending only on $\delta$ and $\varepsilon$, such that

because, by (2.6),

$$
I_{2}<\frac{1}{3} \varepsilon, \quad I_{3}<\frac{1}{3} \varepsilon
$$

$$
I_{2} \leqq \int_{R}^{\infty} e^{\lambda^{\prime \prime}\left(\xi^{\prime}+t-x\right)} d \lambda^{\prime \prime} \leqq \int_{R}^{\infty} e^{-\delta \lambda^{\prime \prime}} d \lambda^{\prime \prime} \leqq \delta^{-1} e^{-\delta R},
$$

and, by (2.13) the same is true for $I_{3}$. The quantity $R$ does not depend on $\Delta t$. Hence, by the comments after $(2.3), \Delta t$ can be chosen so that $I_{1} \leqq \frac{1}{3} \varepsilon$. Hence

$$
\left|A_{t}\left[(x-\xi)^{-1}\right]-\alpha_{t}\left[(x-\xi)^{-1}\right]\right| \leqq I_{1}+I_{2}+I_{3}<\varepsilon .
$$

Because of symmetry, we may replace $x-\xi^{\prime}-\delta$ by $\left|x-\xi^{\prime}\right|-\delta$ in (3.5). 
Thus far, we have assumed that $t$ is positive. However, by symmetry, the results hold for negative $t$, and summing up, we get the following

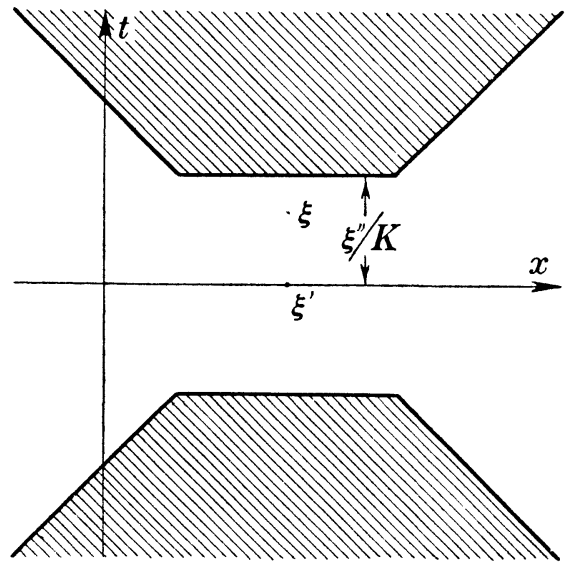

Lemma. $\alpha_{t}\left[(x-\xi)^{-1}\right]$ converges to $A_{t}\left[(x-\xi)^{-1}\right]$ in the real $x t$-plane outside a "shadow" of the pole $\xi$. The "shadow" is determined by the inequalities

$$
|t| \geqq\left|\xi^{\prime \prime}\right| K^{-1}, \quad|t| \geqq\left|x-\xi^{\prime}\right| .
$$

The convergence is uniform in every closed region interior to the unshaded parts of the $\left(x, t, \xi^{\prime}, \xi^{\prime \prime}\right)$-space.

4. The exact shape of the region in the $x t$-plane, in which $\alpha_{t}\left[(x-\xi)^{-1}\right]$ converges to $A_{t}\left[(x-\xi)^{-1}\right]$ has not been found. The Lemma gives sufficient conditions only. One may improve the region slightly by the use of integrals analogous to (3.1) and (3.2) taken from the origin to infinity along different straight lines in the $\lambda$-plane. The following two arguments indicate that we are probably not very far from the best possible region.

First, assume that $\xi$ is real. Then we get, by (3.4) and (2.2) for $x-\xi>u t$,

$\alpha_{t}\left[(x-\xi)^{-1}\right]=\int_{0}^{\infty} e^{-\lambda^{\prime \prime}(x-\xi)} \frac{\cosh \left(\gamma^{\prime \prime}\left(t-\frac{1}{2} \Delta t\right)\right)}{\cosh \frac{1}{2} \gamma^{\prime \prime} \Delta t} d \lambda^{\prime \prime}>\frac{1}{2} \int_{0}^{\infty} e^{-\lambda^{\prime \prime}(x-\xi)+\gamma^{\prime \prime}(t-\Delta t)} d \lambda^{\prime \prime}$.

From (2.12) we can derive a relation of the form

$$
\frac{1}{2} \gamma^{\prime \prime} \Delta t>\frac{1}{2} \lambda^{\prime \prime} \Delta t-A\left(\frac{1}{2} \lambda^{\prime \prime} \Delta t\right)^{3},
$$

where $A$ does not depend on $\Delta t$. Introduce this, and let $u t<x-\xi<t-\Delta t$. Then

$$
\alpha_{t}\left[(x-\xi)^{-1}\right]>\frac{1}{2} \int_{0}^{\infty} e^{-A\left(\frac{1}{2} \Delta t\right)^{2} \lambda^{\prime \prime 3}} d \lambda^{\prime \prime}>C(\Delta t)^{-2 / 3} \rightarrow \infty .
$$

Similar results hold, by symmetry, for $x-\xi<-u t$ and for $t<0$. Furthermore, $\alpha_{t}\left[(x-\xi)^{-1}\right]$ is infinite at the points $(\xi \pm n \Delta x, t)$ in the region $|x-\xi| \leqq u|t|$. (Cf. Section 6.) Hence, when $\xi$ is real, the inequality $|t|<|x-\xi|$ is a necessary and sufficient condition for the convergence of $\alpha_{t}\left[(x-\xi)^{-1}\right]$ to $A_{t}\left[(x-\xi)^{-1}\right]$. 
Second, assume that $\xi^{\prime \prime}<0$. We have, by (3.2) and (2.2),

$$
\alpha_{t}\left[(x-\xi)^{-1}\right]=-i \int_{0}^{\infty} e^{-\lambda\left|\xi^{\prime \prime}\right|} \frac{\cos \gamma\left(t-\frac{1}{2} \Delta t\right)}{\cos \frac{1}{2} \gamma \Delta t} e^{i \lambda\left(x-\xi^{\prime}\right)} d \lambda .
$$

Assume also that $x=\xi^{\prime}$. In the interval

$$
(2 / \Delta x) \arcsin u<\lambda<(2 / \Delta x)(\pi-\arcsin u)
$$

the integrand is given by

$$
-(-1)^{t / \Delta t} e^{-\lambda\left|\xi^{\prime \prime}\right|} \sinh \left(\gamma^{\prime \prime}\left(t-\frac{1}{2} \Delta t\right)\right) / \sinh \frac{1}{2} \gamma^{\prime \prime} \Delta t .
$$

The contribution to the integral from this interval can be estimated by the saddle-point method. The maximum of the integrand is at least as large as its value at that point $\lambda_{m}$, where $\gamma^{\prime \prime} \mid \lambda$ attains its upper bound $K$. Hence the contribution is at least of the order $(\Delta t)^{-\frac{1}{2}} e^{\left(K \lambda_{m} t-\lambda_{m}\left|\xi^{\prime \prime}\right|\right) / \Delta t}$. When $K t>\left|\xi^{\prime \prime}\right|$, the contributions from the remaining parts of the real axis are smaller.

By symmetry, the same holds for $\xi^{\prime \prime}>0$.

Hence, $\alpha_{t}\left[(x-\xi)^{-1}\right]$ diverges on the line $x=\xi^{\prime}$ for $t>\left|\xi^{\prime \prime}\right| / K$. This argument cannot be used, when $x \neq \xi^{\prime}$, because of the oscillating factor $e^{i \lambda\left(x-\xi^{\prime}\right)}$. In that case, the question of getting necessary conditions for convergence is still open.

5. Now, we shall finish the proof of Theorem 1. Assume that $f(\xi)$ is holomorphic within and on the rectangle $R$ defined by the inequalities

$$
a \leqq \xi^{\prime} \leqq b, \quad\left|\xi^{\prime \prime}\right| \leqq \frac{1}{2} K(b-a) .
$$

Then, there exists a contour $C$, enclosing $R$, such that

and

$$
f(x)=-(2 \pi i)^{-1} \int_{C} f(\xi)(x-\xi)^{-1} d \xi
$$

$$
\alpha_{t}[f]=-(2 \pi i)^{-1} \int_{C} f(\xi) \alpha_{t}\left[(x-\xi)^{-1}\right] d \xi .
$$

The "shadows" of the points on $C$ fall entirely in the exterior to the region of determination belonging to the interval $(a, b)$, i.e. the region $a+|t| \leqq x \leqq b-|t|$. We may then apply the Lemma of Section 3. Hence, by the uniform convergence

$$
\lim _{\Delta t \rightarrow 0} \alpha_{t}[f]=-(2 \pi i)^{-1} \int_{\dot{C}}^{0} f(\xi) A_{t}\left[(x-\xi)^{-1}\right] d \xi=A_{t}[f]
$$


for all $(x, t)$ in the region of determination, and the convergence is uniform. After the introduction of the estimates of $K$, given by (2.11), we immediately obtain Theorem 1 .

If the assumptions of regularity for $f(\xi)$ and $g(\xi)$ in Theorem 1 are not satisfied in the whole of the rectangle, associated to the given interval $(a, b)$, the theorem may usually be applied after a division of the interval into smaller parts (which may overlap). Then $\Phi(x, t)$ converges to $F(x, t)$ in the union of the regions of determination of the subintervals. In this way, the Lemma in Section 3 can be obtained as a corollary from the theorem. More generally, this leads to

Theorem 2. When $f(\xi)$ and $g(\xi)$ are analytic, then $\Phi(x, t) \rightarrow F(x, t)$ for all points $(x, t)$ outside the "shadows" of the singularities of $f(\xi)$ and $g(\xi)$.

6. Since most functions of Applied Mathematics are at least piece-wise analytic, one might expect that the theorems just obtained would be more relevant to numerical practice than the negative results of Courant et al. This is, however, not the case (if the difference equation is used for recursive numerical computation of $F(x, t))$. The reason is the presence of round-off errors, which behave very much like non-analyticities. In terms of Fourier analysis, the round-off introduces "wave components", for which $\left|\sin \frac{1}{2} \lambda \Delta x\right|>u$. According to the comments to (2.4), such waves have a very rapid growth. Thus, that circumstance which caused trouble for the proof of convergence under more general assumptions, gives rise to so-called numerical instability in a computation with finite differences.

Even an isolated round-off error has a very strong effect. For $u=\frac{1}{2}$, an error of one unit at $x=x_{0}, t=\Delta t$ propagates according to the following scheme. (Cf. Collatz [1, p. 207 ff.].)

\begin{tabular}{lccrrrr} 
& 0 & $\Delta t$ & $2 \Delta t$ & $3 \Delta t$ & $4 \Delta t$ & \multicolumn{1}{c}{$5 \Delta t$} \\
$x_{0}-4 \Delta x$ & 0 & 0 & 0 & 0 & 0 & 256 \\
$x_{0}-3 \Delta x$ & 0 & 0 & 0 & 0 & 64 & -1536 \\
$x_{0}-2 \Delta x$ & 0 & 0 & 0 & 16 & -288 & 4432 \\
$x_{0}-1 \Delta x$ & 0 & 0 & 4 & -48 & 616 & -7920 \\
$x_{0}$ & 0 & 1 & -6 & 67 & -780 & 9541 \\
$x_{0}+1 \Delta x$ & 0 & 0 & 4 & -48 & 616 & -7920 \\
$x_{0}+2 \Delta x$ & 0 & 0 & 0 & 16 & -288 & 4432 \\
$x_{0}+3 \Delta x$ & 0 & 0 & 0 & 0 & 64 & -1536 \\
$x_{0}+4 \Delta x$ & 0 & 0 & 0 & 0 & 0 & 256
\end{tabular}


In our terminology this function is equal to $\beta_{t}\left[\delta_{x, x_{0}}\right] / \Delta t$, where $\delta_{x, x_{0}}$ is the Kronecker delta. The following facts about the function $\beta_{t}\left[\delta_{x, x_{0}}\right] / \Delta t$ are easily proved by induction, directly from the difference equation, when $u<1$.

1) Its sign has a "chess-board-distribution".

2) Its modulus is at least equal to $u^{-2(t-\Delta t) / \Delta t}$, at every lattice-point $(x, t)$ for which $\left|x-x_{0}\right|<u t$. It follows from (1.5), that the same holds for the function $\varphi\left(\left(x-x_{0}\right) / \Delta x, t\right) \equiv \alpha_{t}\left[\delta_{x, x_{0}}\right]$. In particular $\varphi(\mu, t) \neq 0$, if $|\mu|<t / \Delta t$ and if $\mu$ is an integer, whereas $\varphi(\mu, t)=0$ if $|\mu| \geqq t / \Delta t$. From the identity

$$
f(x)=\sum_{\nu=-\infty}^{\infty} f(\nu \Delta x) \delta_{x, v \Delta x}
$$

it follows that

$$
\alpha_{t}[f(x)]=\sum_{\nu=-\infty}^{\infty} f(v \Delta x) \alpha_{t}\left[\delta_{x, v \Delta x}\right]=\sum_{|\mu|<t / \Delta t} \varphi(\mu, t) f(x+\mu \Delta x) .
$$

This explicit representation of $\alpha_{t}[f]$ is analogous to the expression (1.4) for $A_{t}[f]$. We see from it, for example, that $\alpha_{t}[f(x)]$ is infinite at the points $(\xi+\mu \Delta x, t)$ in the region $|x-\xi|<u t$, if $f(x)$ is infinite at $x=\xi$. (This was stated without proof in Section 4.)

Putting $f(x)=e^{i \lambda x}, x=0$ into (6.1) we obtain a generating function for the weight-coefficients $\varphi(\mu, t)$

$$
\sum_{|\mu|<t / \Delta t} \varphi(\mu, t)\left(e^{i \lambda \Delta x}\right)^{\mu}=\cos \gamma\left(t-\frac{1}{2} \Delta t\right) / \cos \frac{1}{2} \gamma \Delta t .
$$

One hardly expects that $\alpha_{t}[f]$ can be an approximation to $A_{t}[f]$, when looking at (6.1), taking into account the character of $\varphi(\mu, t)$. This fact appears as the result of a cancellation between different large terms, which is made possible by the "smoothness" of analytic functions. We cannot hope for a cancellation of this kind, when round-off errors are present. For instance, consider the initial round-off errors in the values of $f(x)$ as uncorrelated samples from a population with mean zero and variance $\sigma^{2}$, and suppose that there are no new round-off errors in the computations. The first assumption is usually reasonable, although it is very easy to give examples, where it is not valid. The second assumption is more artificial, but it is valid in one special case, namely if $u^{-2}$ is an integer. Then we have for the variance of $\alpha_{t}[f]$

$$
D^{2}\left\{\alpha_{t}[f]\right\}=\sigma^{2} \sum_{\mu}(\varphi(\mu, t))^{2}>\sigma^{2}(2 t / \Delta t-1) u^{-4(t-\Delta t) / \Delta t} .
$$

This indicates that a considerable loss of accuracy is probable, when $u<1$. A sharper estimate may be obtained by the application of Parsevals formula to (6.2). 
From the point of view of discrete Fourier analysis, as used in the investigations of numerical stability by von Neumann, O'Brien et al. [4], Hyman [3], and others, the growth of $\alpha_{t}\left[\delta_{x, x_{0}}\right]$ is due to the considerable intensity of unstable components in the formula

$$
\delta_{x, x_{0}}=N^{-1} \sum_{v=1}^{N} e^{i \lambda_{v}\left(x-x_{0}\right)}, \quad \lambda_{v}=2 \pi v /(b-a), \quad N=(b-a) / \Delta x .
$$

The convergence of $\alpha_{t}[f]$, when $f(x)$ is an analytic, non-periodic function, appears in the discrete Fourier representation as the result of a cancellation between different diverging components. Therefore, the slightly different approach, used in this paper, seems to be more natural.

7. When $u \rightarrow 0$, the equations of Problem $\Delta$ becomes a set of ordinary differential equations

$$
\begin{aligned}
\frac{d^{2} \Phi_{\tau}(x)}{d x^{2}} & =(\Delta t)^{-2}\left(\Phi_{\tau+1}(x)-2 \Phi_{\tau}(x)+\Phi_{\tau-1}(x)\right), \quad \tau=1,2, \ldots, \\
\Phi_{0}(x) & =f(x), \quad \Phi_{1}(x)=f(x)+\Delta t g(x) .
\end{aligned}
$$

According to Theorem 1, the regularity of $f(\xi)$ and $g(\xi)$ in the rectangle

$$
a \leqq \xi^{\prime} \leqq b, \quad\left|\xi^{\prime \prime}\right| \leqq(b-a) / 3
$$

is sufficient for the convergence of $\Phi(x, t)$ to $F(x, t)$ for any positive value of $u$. This indicates that also $\Phi_{t / \Delta t}(x)$ should converge to $F(x, t)$ when $\Delta t \rightarrow 0$. That is true, since the preceding theory can be applied here almost directly with only a few obvious modifications.

Acknowledgement. The author wishes to express his gratitude to the Swedish Board for Computing Machinery, who have made this work possible, and to Dr. N. A. Phillips and Mr. G. Ehrling, who have given valuable advice after reading the manuscript.

\section{REFERENCES}

1. L. Collatz, Numerische Behandlung von Differentialgleichungen, Berlin, 1951.

2. R. Courant, K. Friedrichs und H. Lewy, Uber die partiellen Differenzengleichungen der mathematischen Physik, Math. Ann. 100 (1928), 32-74.

3. M. A. Hyman, On the numerical solution of partial differential equations, Diss., Delft, 1953.

4. G. G. O'Brien, M. A. Hyman, and S. Kaplan, A study of the numerical solution of partial differential equations, Journ. Math. Phys. 29 (1951), 223-251.

5. E. C. Titchmarsh, Introduction to the theory of Fourier integrals, Oxford, 1937. 